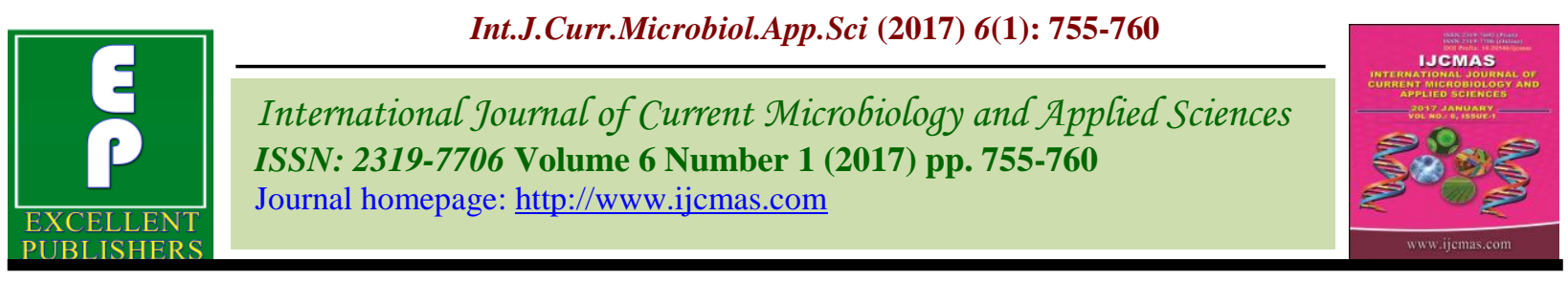

Original Research Article

http://dx.doi.org/10.20546/ijcmas.2017.601.090

\title{
Role of CT-Scan versus Chest X-RAY in the Diagnosis of Community- Acquired Pneumonia
}

\author{
Eliane El-Sarraf ${ }^{1}$, Nayla Nicolas ${ }^{1}$, Souheil Hallit ${ }^{1,2,3,4 *}$ and Rabih Hallit ${ }^{1}$ \\ ${ }^{1}$ Universite Saint-Esprit Kaslik, Faculty of Medicine, Kaslik, Lebanon \\ ${ }^{2}$ Psychiatric Hospital of the Cross, Jal Eddib, Lebanon \\ ${ }^{3}$ Lebanese University, School of Pharmacy, Beirut, Lebanon \\ ${ }^{4}$ Universite Saint Joseph, Faculty of Pharmacy, Beirut, Lebanon \\ *Corresponding author
}

\section{A B S T R A C T}

\section{Keywords \\ Community- \\ acquired \\ pneumonia; CT- \\ scan; Chest X-ray; infiltrates. \\ Article Info \\ Accepted: \\ 29 December 2016 \\ Available Online: \\ 10 January 2017}

Until now, there is no adequate definition of specific indications of an early and priority request for chest CT. To reveal and identify the number of community acquired pneumonia (CAP) under diagnosed by chest X-ray (CXR) and uncovered by CT-scan (CTS) in Lebanese hospitalized adult patients. A cross-sectional study was conducted and a total sample of 101 patients with CAP was recruited, with all patients having undergone CXR and chest CTS for suspected cases of pneumonia. CXRs and CTS, performed in the same patient, were matched. Pulmonary infiltrates were seen on chest X-ray in $62(61.4 \%)$ patients. In contrast, 39 (38.6\%) of patients had infiltrates on the chest CTS without having retrospectively any infiltrates on the CXR. Patients aged $\leq 55$ years with cough $(n=13$, $81.3 \%$ ) the chest $\mathrm{X}$-ray was significantly sufficient to diagnose pneumonia as compared to CT-scan $(\mathrm{p}=0.028)$ while patients $>55$ years with pulmonary disease seem to be in favor of an early CT-scan in order to reveal their true condition $(25 \%, \mathrm{p}=0.040)$. Taking a look at patients aged more than 55 years, those having myalgia and hemoptysis $(n=6,13 \%)$ did not require more than a simple $\mathrm{X}$-ray to distinguish the infiltrates $(\mathrm{p}=0.026)$. Patients less than or equal to 55 years with pulmonary disease and smoking patients, regardless their age, require and may benefit from an early CTS.

\section{Introduction}

Pneumonia is a lower respiratory infection that affects the alveoli and is considered as a major socio-economic burden.

"Dr. William Osler, the founder of modern me dicine, who has studied pneumonia throughout his career, called pneumonia, the captain of the men of death", due to its immeasurable havoc and burdens on humanity (Mizgerd, 2008).
In 1930, before the revolution of antibiotics, pneumonia was the third cause of mortality in the US (Country Analysis Report, 2017). In 2015, 922000 children under age of five died from complicated pneumonia which constitutes 15 percent of deaths recorded among this category worldwide (Country Analysis Report, 2017). This comparison clearly shows the persistence of pneumonia as one of the major causes of 
mortality of children and adults in the world. Therefore, antibiotic era resulted only in 5\% decrease in mortality (Country Analysis Report, 2017).

Community acquired pneumonia is usually detected in patients presenting to the emergency department (Dorca et al., 1996). Delaying targeted and adequate treatment leads to an increasingly severe and obscure prognosis, hence the importance to make an early diagnosis (Houck et al., 2004). Clinical and biological community acquired pneumonia (CAP) diagnosis are inconclusive, therefore parenchymal infiltrates on chest Xray are the evidence of existing pneumonia (Metlay et al., 1997). Moreover, the appearance of the infiltrates on the radiography is sometimes delayed and even the quality is altered because of the underlying pathologies, which makes the chest X-ray a major but imperfect diagnostic tool in CAP. Chest X-ray remains the simplest exam and the most frequently used to diagnose and assess the extent of pneumonia. Its strict interpretation remains a key consideration (Lim et al., 2010).

Some references summarize the role of CTscan as a conclusive means when the interpretation of Chest X-ray remains difficult, while other authors argue the need of an early CT-scan in order to improve the diagnostic sensitivity of pneumonia. However, in many situations a typical clinical picture of pneumonia is associated with a normal chest X-Ray while the CT-Scan is positive and vice versa leading respectively to underestimation of pneumonia with delaying adequate therapy and over-prescription of antibiotics.

The aim of this study is to reveal and identify the number of CAP under diagnosed by CXR and uncovered by CT-scan in Lebanese hospitalized adult patients.

\section{Methods}

This cross-sectional study was conducted between June 2015 and August 2016. Cases were chosen from the University hospital center Notre-Dame de Secours, located in Jbeil, Lebanon. A total sample of 101 patients with community acquired pneumonia was recruited, with all patients having undergone CXR and chest CT- Scan for suspected cases of pneumonia. CXRs and CT scans, performed in the same patient, were matched.

Patients were included in the study if they were 18 years and above, hospitalized patients and patient with infiltrates on CT-scan and have done a Chest X-Ray. Moreover, pregnant and breastfeeding women, or with a medical history of health care associated infections or had a chest CT-scan performed after 24 hours of the chest X-ray were excluded.

\section{Ethical aspect}

The Institutional Review Board of the hospital waived the need for an approval based on the facts that it was an observational study that respected participants' autonomy and confidentiality and induced minimal harm to them.

\section{Questionnaire and variables}

We studied the parameters that may affect the radiologic delay of infiltrates, based on the United States Infectious Disease Society/United States Thoracic Society "Guide to the Diagnosis and Treatment of Community- Acquired Pneumonia in Adults (2007)".Consequently, the variables were classified into sociodemographic characteristics (age and gender), presence of one or more of the comorbidities (diabetes, kidney disease, liver disease, history of stroke, congestive heart failure, respiratory diseases and neoplasia), previous antibiotic 
treatment, signs and symptoms upon physical exam (cough, chest pain, sputum production, dyspnea, respiratory rate $>30 / \mathrm{min}$, crackles, chills, myalgia, fever, confusion, heart rate $>125 / \mathrm{min}$, systolic blood pressure < 90 $\mathrm{mmHg}$, diastolic blood pressure $<60 \mathrm{mmHg}$ ), biologic data (white blood cell count $>10 \times 10^{9} / \mathrm{L}$ or $<4 \times 10^{9} / \mathrm{L}$, with or without an accompanying shift to the left, C-reactive protein (CRP) and finally radiological data.

\section{Statistical analysis}

Descriptive statistics were calculated for all study variables. This includes the mean and standard deviation for continuous measures, counts and percentages for categorical variables. Paired t-tests were used to look for difference between. The statistical package SPSS version 22 was used for all statistical analysis. Statistical significance was set at $\mathrm{p}<$ 0.05.Paired sample t-test was used for bivariate analysis to check a significant difference between chest X-ray and CT-scan results.

\section{Results and Discussion}

\section{Chest X-ray results and CT scan results}

Pulmonary infiltrates were seen on chest Xray in $62(61.4 \%)$ patients. In contrast, 39 (38.6\%) of patients had infiltrates on the chest CT-scan without having retrospectively any infiltrates on the chest X-ray $(\mathrm{p}<0.001)$.

\section{Study of parameters}

Sex ratio was approximately 1 . The majority of the patients $(81 \%)$ were 55 years of age or older.

Cough $(\mathrm{n}=59,58.41 \%)$ and dyspnea $(\mathrm{n}=52$, $51.48 \%$ ) were the most frequent symptoms, with myalgia and hemoptysis being the least common ones. Characteristics of the location of pneumonia were not significant with no preference of Chest X-ray over CT-Scan and vice versa.

In addition, $55.44 \%$ of patients with CAP were smokers, $66.7 \%(\mathrm{p}=0.042)$ of whom had normal chest X-Ray with positive CT-scan.

Radiography in decubitus or lateral position didn't affect the sensitivity of CXR in comparison with CT-Scan $(\mathrm{p}=0.576)$.

In patients whose age is less than or equal to 55 years with cough $(n=13,81.3 \%)$ the chest $\mathrm{X}$-ray was significantly sufficient to diagnose pneumonia as compared to CT-scan $(\mathrm{p}=0.028)$.

On the other hand, patients whose age was less than or equal to 55 years with pulmonary disease, seem to be in favor of an early CTscan in order to reveal their true condition $(25 \%, \mathrm{p}=0.040)$.

Taking a look at patients aged more than 55 years, those having myalgia and hemoptysis $(n=6,13 \%)$ did not require more than a simple $\mathrm{X}$-ray to distinguish the infiltrates $(\mathrm{p}=0.026)$.

Our findings revealed that 39 out of 101 patients had a normal radiography as well as a positive CT-scan in favor of pneumonia. At the end, the aim of our study was to identify number of CAP missed on Chest X-Ray and discovered on CT-scan. Our findings revealed that pneumonias were significantly missed on the X-Ray, while being present on the CTscan. This result meets the recent findings of Samuel Nadler which highlighted a $33.33 \%$ of missed infiltrates.

Considering the ultimate importance of early diagnosis in decreasing the mortality rate and increasing the response to antibiotics, clinicians should rely more on CT-scan in specific conditions. 
Despite all the efforts and the revolutions done on antibiotics, pneumonia remains a leading cause of mortality and a major socioeconomic problem. Early diagnosis and adequate antibiotic therapy constitute the cornerstone in decreasing the morbidity and mortality of this disease.
In this study, we redefined CAP as an illness of all ages, favoring patients more than or equal to 55 years with no sex ratio significance. Our results showed that most patients with CAP have cough and dyspnea simultaneously, in line with the results of Le Bel et al., (2015).

Table.1 Bivariate analysis of the factors affecting the conformity of the Chest X-ray versus CT-scan

\begin{tabular}{|c|c|c|c|c|}
\hline & & Cor & rmity & $P$-value \\
\hline & & $\mathbf{0}$ & 1 & \\
\hline Position while & Standing & $23(59.0 \%)$ & $40(64.5 \%)$ & 0.576 \\
\hline performing CRX/ CTS & In bed & $16(41.0 \%)$ & $22(35.5 \%)$ & \\
\hline Cough & 0 & $20(51.3 \%)$ & $22(35.5 \%)$ & 0.117 \\
\hline & 1 & $19(48.7 \%)$ & $40(64.5 \%)$ & \\
\hline Sputum & 0 & $22(56.4 \%)$ & $38(61.3 \%)$ & 0.627 \\
\hline & 1 & $17(43.6 \%)$ & $24(38.7 \%)$ & \\
\hline Myalgia & 0 & $39(100 \%)$ & $55(88.7 \%)$ & 0.041 \\
\hline & 1 & $0(0.0 \%)$ & $7(11.3 \%)$ & \\
\hline Dyspnea & 0 & $20(51.3 \%)$ & $29(46.8 \%)$ & 0.659 \\
\hline & 1 & $19(48.7 \%)$ & $33(53.2 \%)$ & \\
\hline Hemoptysis & 0 & $39(100 \%)$ & $55(88.7 \%)$ & 0.041 \\
\hline & 1 & $0(0.0 \%)$ & $7(11.3 \%)$ & \\
\hline Pulmonary disease & 0 & $25(64.1 \%)$ & $48(77.4 \%)$ & 0.145 \\
\hline & 1 & $14(35.9 \%)$ & $14(22.6 \%)$ & \\
\hline Smoking & 0 & $13(33.3 \%)$ & $32(51.6 \%)$ & 0.042 \\
\hline & 1 & $26(66.7 \%)$ & $30(48.4 \%)$ & \\
\hline Right side & 0 & $22(56.4 \%)$ & $38(61.3 \%)$ & 0.627 \\
\hline & 1 & $17(43.6 \%)$ & $24(38.7 \%)$ & \\
\hline Left side & 0 & $27(69.2 \%)$ & $50(80.6 \%)$ & 0.189 \\
\hline & 1 & $12(30.8 \%)$ & $12(19.4 \%)$ & \\
\hline Bilateral & 0 & $29(74.4 \%)$ & $36(58.1 \%)$ & 0.096 \\
\hline & 1 & $10(25.6 \%)$ & $26(41.9 \%)$ & \\
\hline Rapid flu test & 0 & $38(97.4 \%)$ & $57(91.9 \%)$ & 0.401 \\
\hline & 1 & $1(2.6 \%)$ & $5(8.1 \%)$ & \\
\hline Age less or equal 55 & 0 & $35(89.7 \%)$ & $46(74.2 \%)$ & 0.056 \\
\hline & 1 & $4(10.3 \%)$ & $16(25.8 \%)$ & \\
\hline Age more than 55 & 0 & $4(10.3 \%)$ & $16(25.8 \%)$ & 0.046 \\
\hline & 1 & $35(89.7 \%)$ & $46(74.2 \%)$ & \\
\hline Gender & 1 & $19(48.7 \%)$ & $32(51.6 \%)$ & 0.777 \\
\hline & 2 & $20(51.3 \%)$ & $30(48.4 \%)$ & \\
\hline
\end{tabular}


Table.2 Stratified analysis of the factors affecting the conformity of the Chest X-ray versus CT-scan based on age.

\begin{tabular}{|c|c|c|c|c|c|c|c|}
\hline & \multirow{2}{*}{\multicolumn{2}{|c|}{$\begin{array}{c}\text { Age } \leq 55 \text { years } \\
\text { Conformity }\end{array}$}} & \multicolumn{4}{|c|}{ Age $>55$ years } \\
\hline & & & & \multirow{2}{*}{ P -value } & \multicolumn{2}{|c|}{ Conformity } & \multirow[t]{2}{*}{$\mathbf{P}$-value } \\
\hline & & 0 & 1 & & 0 & 1 & \\
\hline \multirow[t]{2}{*}{ Cough } & $\mathbf{0}$ & $3(75.0 \%)$ & $3(18.8 \%)$ & \multirow[t]{2}{*}{0.028} & $17(48.6 \%)$ & $19(41.3 \%)$ & \multirow[t]{2}{*}{0.514} \\
\hline & 1 & $1(25.0 \%)$ & $13(81.3 \%)$ & & $18(51.4 \%)$ & $27(58.7 \%)$ & \\
\hline \multirow[t]{2}{*}{ Myalgia } & $\mathbf{0}$ & $4(100 \%)$ & $15(93.8 \%)$ & \multirow[t]{2}{*}{0.608} & $35(100 \%)$ & $40(87.0 \%)$ & \multirow[t]{2}{*}{0.026} \\
\hline & 1 & $0(0.0 \%)$ & $1(6.3 \%)$ & & $0(0.0 \%)$ & $6(13.0 \%)$ & \\
\hline \multirow{2}{*}{ Hemoptysis } & $\mathbf{0}$ & $4(100 \%)$ & $15(93.8 \%)$ & \multirow[t]{2}{*}{0.608} & $35(100 \%)$ & $40(87.0 \%)$ & \multirow{2}{*}{0.026} \\
\hline & 1 & $0(0.0 \%)$ & $1(6.3 \%)$ & & $0(0.0 \%)$ & $6(13.0 \%)$ & \\
\hline \multirow{4}{*}{$\begin{array}{l}\text { Pulmonary } \\
\text { disease } \\
\text { Right side }\end{array}$} & $\mathbf{0}$ & $3(75.0 \%)$ & $16(100 \%)$ & \multirow[t]{2}{*}{0.040} & $22(62.9 \%)$ & $32(69.6 \%)$ & \multirow[t]{2}{*}{0.526} \\
\hline & 1 & $1(25.0 \%)$ & $0(0.0 \%)$ & & $13(37.1 \%)$ & $14(30.4 \%)$ & \\
\hline & $\mathbf{0}$ & $1(25.0 \%)$ & $12(75.0 \%)$ & \multirow[t]{2}{*}{0.061} & $21(60.0 \%)$ & $26(56.5 \%)$ & \multirow[t]{2}{*}{0.753} \\
\hline & 1 & $3(75.0 \%)$ & $4(25.0 \%)$ & & $14(40.0 \%)$ & $20(43.5 \%)$ & \\
\hline \multirow[t]{2}{*}{ Left side } & $\mathbf{0}$ & $4(100 \%)$ & $12(75.0 \%)$ & \multirow{2}{*}{0.264} & $23(65.7 \%)$ & $38(82.6 \%)$ & \multirow[t]{2}{*}{0.081} \\
\hline & 1 & $0(0.0 \%)$ & $4(25.0 \%)$ & & $12(34.3 \%)$ & $8(17.4 \%)$ & \\
\hline
\end{tabular}

According to our findings, patients less than or equal to 55 years with pulmonary disease require an early CT-scan, in the same context Deepak Garg and al, noted that CT-scan were performed more in younger patients (Deepak et al., 2014). In addition, smoking patients regardless of their age, require an early $\mathrm{Ct}$ scan.

Chest X-Ray plays a major role in the diagnosis of pneumonia, however chest CTscan is more sensitive. There are few studies that highlight the benefit of chest CT-scan in the context of adult patients having pneumonia; Nyamande and al demonstrated, in $40 \mathrm{HIV}$ patients with normal X-ray findings, that $82 \%$ had abnormal CT-scan. In addition, Beal and al showed that CT-scan uncovered missing findings on previous $\mathrm{X}$ rays in immunocompromised as well as immunocompetent patients, which induced in $40 \%$ of cases modifications in the management (Beall et al., 1998).

Yann-Erick and al, concluded his study by asserting that $33 \%$ of CAP - suspected patients, with negative chest X-ray, has been diagnosed on CT-scan, the latter markedly affecting both diagnosis and clinical management of CAP. The important role as well as the high sensitivity offered by the chest CT-scan is no longer to be discussed. Thus, our study is a first step in an array of future research that we must carry out in order to define the indications of the thoracic CTscan depending on particular factors.

\section{Limitations}

This study has limitations. First of all, our sample is considered a small sample. Further larger studies are needed to help correlate more parameters with early CT-scan and to consolidate our results. A selection bias is possible since the study was limited to one specific hospital setting.

In conclusion, in our study we have restored what has been demonstrated by the other researches and we tried to emphasize on the parameters that affect this discrepancy between chest radiography and CT scan. According to our findings, patients less than or equal to 55 years with pulmonary disease and smoking patients, regardless their age, require and may benefit from an early CT- 
scan. Our study is a seed sown in a fertile research field, prompting public health officials, to conduct prospective studies in order to dissolve this dilemma and to define new guidelines leading to a unique diagnostic and therapeutic approach as well as a solid confrontation of "the captain of the men of death".

\section{References}

Beall, D.P., Scott, W.W. Jr, Kuhlman, J.E., Hofmann, L.V., Moore, R.D., Mundy, L.M. 1998. Utilization of computed tomography in patients hospitalized with communityacquired pneumonia. Md. Med. J., 47(4):182-187.

Claessens, Y.E., Debray, M.P., Tubach, F., et al. 2015. Early chest computed tomography scan to assist diagnosis and guide treatment decision for suspected community-acquired pneumonia. Am. J. Respir. Crit. Care Med., 192: 974-982.

CorralesMedina, V.F., Musher, D.M., Wells, G.A. , Chirinos, J.A., Chen, L., Fine, M.J. 2012.

Cardiac complications in patients with community-acquired pneumonia: incidence, timing, risk factors, and association with short-term mortality. Circulation, 125:773781.

Country Analysis Report. 2017. Lao PDR Analysis to inform the Lao People's Democratic Republic- United Nations Partnership Framework (2017-2021)

Deepak Garg, Leonard, B., Johnson, Susan Szpunar, Joel, T., Fishbain. 2014. Clinical value of chest computerized tomography scans in patients admitted with pneumonia, J. Hospital Med., 9: 447-450, DOI: $10.1002 / \mathrm{jhm} .2190$

Dorca, J., Torres, A. 1996. Lower respiratory tract infections in the community: towards a more rational approach. Eur. Respir. J., 9: 1588-1589.

Houck, P.M., Bratzler, D.W., Nsa, W., Ma, A, Bar tlett, J.G. 2004. Timing of antibiotic administration and outcomes for Medicare patients hospitalized with communityacquired pneumonia. Arch. Intern. Med., 164:637-644.

Le Bel, et al. 2015. Diagnostic accuracy of Creactive protein and procalcitonin in suspected community-acquired pneumonia adults visiting emergency department and having a systematic thoracic CT scan Critical Care (2015) 19:366 DOI 10.1186/s13054-015-1083-6.

Lim, W.S., Levy, M.L., Macfarlane, J.T.2010.

British Thoracic Society Community Acquired Pneumonia Guidelines Committee. Community acquired pneumonia. Management in primary care. BMJ, 341: c4469.

Metlay, J.P., Schulz, R., Li, Y.H., Singer, D.EMar rie, T.J., Coley, C.M., Hough, L.J., Obrosky , D.S., Kapoor, W.N., Fine, M.J.

1997. Influence of age on symptoms at presentation in patients with communityacquired pneumonia. Arch Intern. Med., 157:1453-1459.

Mizgerd, J.P. Acute lower respiratory tract infection. N. Engl. J. Med., 358: 716-727.

Nyamande, K., Lalloo, U.G., Vawda, F. 2007. Comparison of plain chest radiography and high-resolution CT in human immunodeficiency virus infected patients with community-acquired pneumonia: a sub-Saharan Africa study. Br. J. Radiol., 80(953): 302-306.

Syrjälä, H., Broas, M., Suramo, I., Ojala, A., Lähd e, S. 1998. High-resolution computed tomography for the diagnosis of community-acquired pneumonia. Clin. Infect. Dis., 27: 358-363.

\section{How to cite this article:}

Eliane El-Sarraf, Nayla Nicolas, Souheil Hallit, Rabih Hallit. 2017. Role of CT-Scan versus Chest X-RAY in the Diagnosis of Community-Acquired Pneumonia. Int.J.Curr.Microbiol.App.Sci. 6(1): 755-760. doi: http://dx.doi.org/10.20546/ijcmas.2017.601.090 